\title{
Effect of diethylcarbamazine on HIV load, CD4\% and CD4/CD8 ratio in HIV-infected adult Tanzanians with or without lymphatic filariasis randomized double-blind and placebo-controlled cross-over trial
}

Nielsen, Nina O.; Simonsen, Paul Erik; Dalgaard, Peter; Krarup, Henrik; Magnussen, Pascal; Magesa, Stephen; Friis, Henrik

Published in:

American Journal of Tropical Medicine and Hygiene

Publication date:

2007

Document version

Publisher's PDF, also known as Version of record

Citation for published version (APA):

Nielsen, N. O., Simonsen, P. E., Dalgaard, P., Krarup, H., Magnussen, P., Magesa, S., \& Friis, H. (2007). Effect of diethylcarbamazine on HIV load, CD4\% and CD4/CD8 ratio in HIV-infected adult Tanzanians with or without lymphatic filariasis: randomized double-blind and placebo-controlled cross-over trial. American Journal of Tropical Medicine and Hygiene, 77(3), 507-513. http://www.ajtmh.org/cgi/content/abstract/77/3/507 


\title{
Effect of Diethylcarbamazine on HIV Load, CD4\%, and CD4/CD8 Ratio in HIV-Infected Adult Tanzanians with or without Lymphatic Filariasis: Randomized Double-Blind and Placebo-Controlled Cross-Over Trial
}

\begin{abstract}
Nina O. Nielsen, * Paul E. Simonsen, Peter Dalgaard, Henrik Krarup, Pascal Magnussen, Stephen Magesa, and Henrik Friis DBL-Centre for Health Research and Development, University of Copenhagen, Copenhagen, Denmark; Department of Biostatistics, University of Copenhagen, Copenhagen, Denmark; Department of Clinical Biochemistry, Aalborg University Hospital, Aalborg, Denmark; National Institute for Medical Research, Ubwari Research Station, Tanzania; Department of Human Nutrition, University of Copenhagen, Copenhagen, Denmark
\end{abstract}

\begin{abstract}
We assessed the effect of anti-filarial treatment (diethylcarbamazine, DEC) on HIV load, CD4\%, and CD4/CD8 ratio in HIV-positive individuals with and without infection with the filarial parasite Wuchereria bancrofti in a randomized, double-blind, placebo-controlled cross-over trial. The study was conducted in Tanga Region, Tanzania, in 2002 and involved 27 adults. A significant decrease in HIV load (54\%) and an insignificant increase in CD4\% were observed in the HIV-positive individuals with filarial co-infection at 12 weeks after treatment. HIV load and CD4\% both increased, although not statistically significantly, in the HIV-positive individuals without filarial infection. The findings suggest that DEC affected HIV load through its effect on the filarial infection rather than through a direct (pharmacodynamic) effect on HIV. Global efforts to control lymphatic filariasis by annual mass treatment with DEC may have a beneficial effect on the HIV/AIDS epidemic in areas where HIV and lymphatic filariasis co-exist.
\end{abstract}

\section{INTRODUCTION}

The high prevalence of HIV and parasite infections in subSaharan Africa results in large geographical overlaps and thereby potential for interaction between these two types of infections in co-infected individuals. Parasites, helminths in particular, may increase susceptibility to HIV and contribute to a more rapid progression toward AIDS. ${ }^{1-3}$ Helminthinduced immune activation may be an underlying mechanism, as helminth infections are associated with increased viral replication. ${ }^{3}$ Th1-Th2 cross-regulation is likely to play a role in this interaction. Helminth infections are commonly associated with an upregulated Th2 response. This seems to prevent production of Th1-related cytokines and cytotoxic T-cell activity, ${ }^{1,4}$ which play a central role in the immunologic combat against HIV. HIV virus may furthermore replicate more rapidly in Th2 cells than in Th1 cells, ${ }^{5}$ and a significant level of replication seems to occur in eosinophils in individuals with helminth-induced eosinophilia. ${ }^{6}$ Treatment of helminth infections is thus likely to be particularly beneficial to HIV patients and has been found to be associated with a significant decrease in HIV plasma load. ${ }^{7}$ However, it has also been observed that helminth infections were not associated with faster progression of HIV disease, and it was suggested that anthelminthic therapy may not slow down HIV progression in co-infected individuals. ${ }^{8}$ Apart from helminth infections, some protozoan infections interact with HIV. Thus, malaria has been shown to be an important factor in promoting the spread of HIV in sub-Saharan Africa. ${ }^{9}$

We previously examined the cross-sectional relationship between subclinical HIV, lymphatic filariasis (Wuchereria bancrofti infection), and other parasitoses (Plasmodium falciparum, Ascaris lumbricoides, Trichuris trichiura, and hookworm infections) among 858 adults in Tanga Region, northeastern Tanzania. ${ }^{10}$ A significant positive association was ob-

* Address correspondence to Nina O. Nielsen, DBL-Centre for Health Research and Development, Department of Veterinary Pathobiology, Faculty of Life Sciences, University of Copenhagen, Jægersborg Allé 1D, 2920 Charlottenlund, Denmark. E-mail: nnielsen@dblnet.dk served between HIV and $W$. bancrofti infection and between HIV and $P$. falciparum infection. By contrast, HIV and hookworm infection were negatively associated. A more detailed immunologic study carried out in a subpopulation of 59 individuals, however, could not provide clear evidence for an interaction between HIV and $W$. bancrofti infection. ${ }^{11}$

In the present study, we looked further into the association between HIV and W. bancrofti infection, by analyzing the effect of diethylcarbamazine (DEC) treatment of W. bancrofti on HIV infection in HIV-positive individuals with $W$. bancrofti co-infection and compared this with the effect in HIVpositive individuals without filarial co-infection.

\section{MATERIALS AND METHODS}

Study population and study design. The study was conducted to assess and compare the effect of DEC on viral load, CD4\%, and CD4/CD8 ratio in HIV-positive individuals with or without $W$. bancrofti co-infection in a randomized, doubleblind, placebo-controlled cross-over trial. The study was carried out in May-November 2002 in 5 villages (Mwahako, Mwakidila, Pongwe South, Kakindu, and Jitengeni) in Tanga Region, northeastern Tanzania. As part of a cross-sectional study on the association between HIV, lymphatic filariasis, and other parasitic infections, 858 adults (18-70 years) were tested for HIV (antibodies), lymphatic filariasis (W. bancrofti circulating filarial antigens, CFA), malaria parasites, and intestinal helminth eggs. ${ }^{10}$ Pre- and post-test counseling was provided by the Tanga AIDS Working Group, a local NGO. The 858 individuals constituted the source of study individuals for further detailed investigations on HIV and filariasis interactions.

Thirty-four individuals were willing to participate in the current study and met the inclusion criteria of being 1) HIVpositive and 2) without obvious clinical manifestations of HIV and/or $W$. bancrofti infection. The study individuals were randomly assigned to receive DEC or placebo at the beginning of the study, and the opposite treatment was given 12 weeks later (Figure 1). Venous blood was collected at baseline and at $1,12,13$, and 24 weeks after the first round of treatment. Seven of the 34 participants did not complete all follow-up 


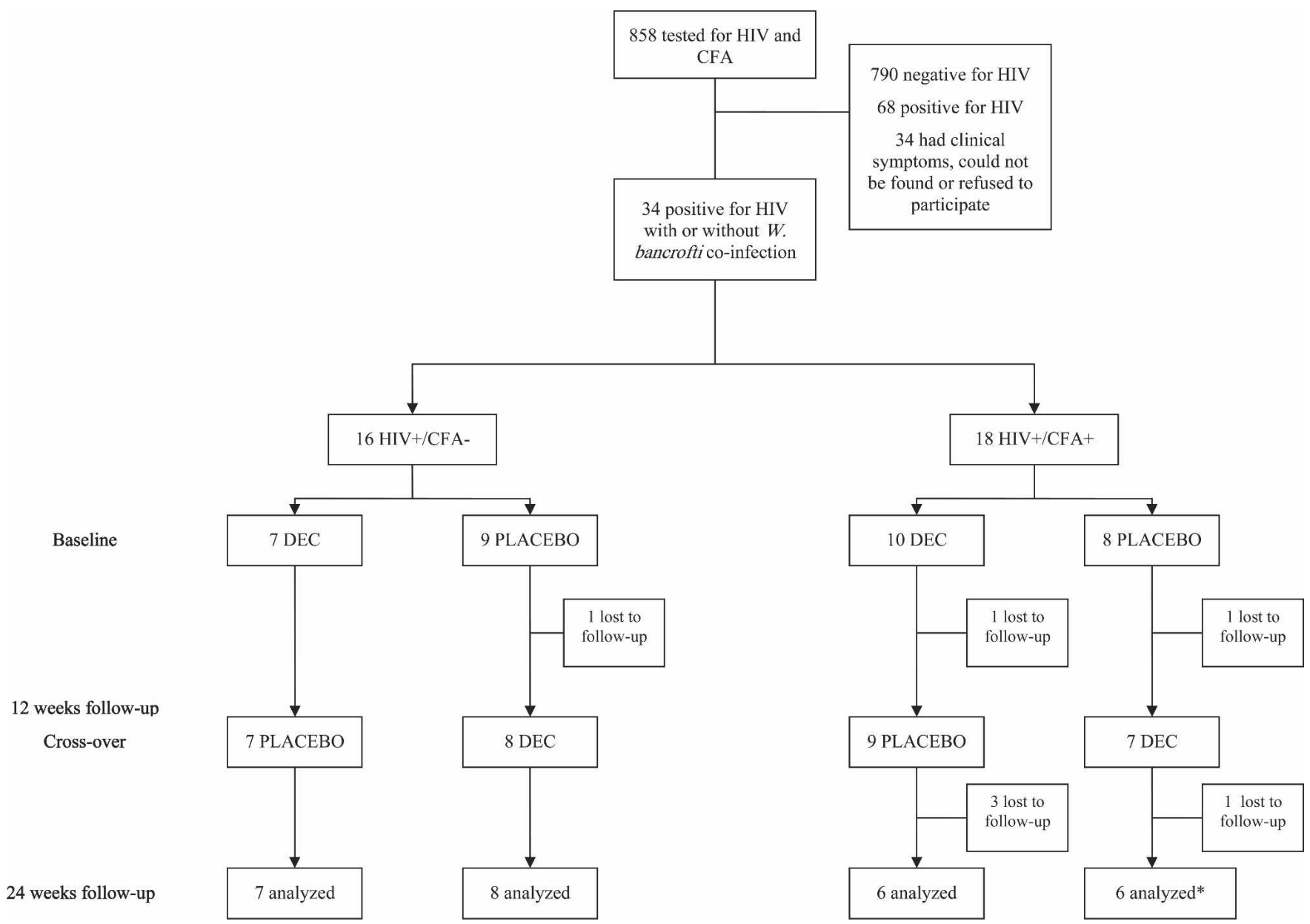

FIGURE 1. Study profile showing details of treatment with diethylcarbamazine (DEC) and placebo. Losses to follow-up were due mainly to participants' wish to withdraw from the study or absence at follow-up. $\mathrm{HIV}^{+}$, HIV-positive individuals; $\mathrm{CFA}^{+}$, individuals positive for $W$. bancrofti CFA; $\mathrm{CFA}^{-}$, individuals negative for $W$. bancrofti CFA. *For HIV load analyses, only 5 were analyzed because the HIV RNA could not be amplified from 1 sample.

examinations and were therefore excluded from the analyses. There was no difference in age, sex, or infections between those lost to follow-up and those who remained in the study. Data from 27 individuals are analyzed. We tested the hypothesis that DEC treatment would 1) reduce the viral load, 2) increase the $\mathrm{CD} 4 \%, 3$ ) increase the $\mathrm{CD} 4 / \mathrm{CD} 8$ ratio, and 4) affect HIV-positive individuals with or without $W$. bancrofti co-infection differently. Research clearance to conduct the study was obtained from the Medical Research Coordinating Committee of the National Institute for Medical Research in Tanzania, and the study was approved by the Danish National Committee on Biomedical Research Ethics.

Treatment with diethylcarbamazine (DEC). The 34 study participants were treated with DEC either at the beginning of the study or after 12 weeks. The drug was administered as a single dose of $6 \mathrm{mg} / \mathrm{kg}$ body weight, which is the yearly dose given for transmission control of lymphatic filariasis. The tablets were prepared specifically for this study by a manufacturer (Almega, Ringsted, Denmark); 50-mg DEC tablets and corresponding placebo tablets were supplied. The identical DEC and placebo tablets were packed in containers with different color codes ("red" or "blue"). Individuals were randomized (1:1) to receive treatment in the order of "red" followed by "blue" or "blue" followed by "red" by using a list of random numbers. ${ }^{12}$ The selected study participants were listed and numbered from 1 to 34 , and the first 17 numbers (between 1 and 34) encountered in the table (when starting on a randomly chosen figure) were assigned to receive treatment in the order red-blue, and the remaining 17 received treatment in the order blue-red. All study personnel and participants were blinded to treatment assignment throughout the study.

Venous blood samples. At each sampling time, $25 \mathrm{~mL}$ of venous blood $(20 \mathrm{~mL}$ heparinized and $5 \mathrm{~mL}$ non-heparinized) was collected from each study participant. The blood was transferred in a cool box to the field laboratory. The $5 \mathrm{~mL}$ of nonheparinized blood was left overnight at $4^{\circ} \mathrm{C}$ to clot. The serum was collected the following day and kept at $-80^{\circ} \mathrm{C}$ until used for quantitative determination of HIV load and CFA intensity. The $20 \mathrm{~mL}$ of heparinized blood was used for isolation of peripheral blood mononuclear cells (PBMCs) and for cell culturing, separation of plasma, hemoglobin measurements, and preparation of blood slides for further immunologic studies.

Circulating filarial antigen (CFA). The W. bancrofti CFA status was determined in the field by use of the NOW immunochromatographic test (ICT) for filariasis (Binax, Scarborough, ME), a rapid, qualitative immunodiagnostic test, as de- 
scribed previously. ${ }^{10}$ The intensity of CFA was later quantified in serum using the TropBio ELISA test kit (TropBio Pty. Ltd., Townsville, Australia). Manufacturers' procedures were followed, and all tests were carried out in duplicate. Serum samples with a mean optical density (OD) value $>$ standard 2 (32 antigen units) were considered positive. Most positive samples had a mean OD value $\geq$ that of the highest standard (standard $7=32,000$ antigen units). These were subsequently diluted 1:10 and retested. Samples with a mean OD value $\geq$ that of the highest standard after dilution $(N=11)$ were assigned a value of 320,000 antigen units (corresponding to 32,000 antigen units multiplied by the dilution factor 10 ).

Assessment of HIV infection. HIV status was determined in the field by 2 different rapid tests (both detecting antibodies to HIV 1 and 2), as described previously. ${ }^{9}$ The HIV load in samples from individuals tested positive in both rapid tests (discrepancies did not occur) was quantified using a modification of an in-house developed reverse-transcriptase PCR. ${ }^{13}$ HIV RNA was isolated from $125 \mu \mathrm{L}$ of serum. All samples were analyzed in 2 independent extractions and runs. Samples with discordant results (discrepancy $>0.5 \mathrm{log}$ ) were retested in duplicate. The limit of detection was 40 genome equivalents (geq) $/ \mathrm{mL}$.

Staining and counting of CD4 and CD8 T cells. An immunoalkaline (IA) phosphatase method was used for staining and counting of CD4 and CD8 T cells. ${ }^{14-16}$ In brief, 2 thin smears from each patient underwent immunocytochemical labeling with monoclonal antibodies against CD4 and CD8 receptors, respectively. The strengthened (by a secondary antibody conjugated to biotin and an avidin-biotin complex conjugated to alkaline phosphatase, both from DakoCytomation, Carpinteria, CA) antigen/antibody binding was visualized as a reddish-stained ring around the lymphocyte nucleus by adding a substrate containing New Fuchsin (fuchsin + substratechromogen system, DakoCytomation). Stained CD4 and CD8 cells were counted among 200 lymphocytes, and the CD4\% and the CD4/CD8 ratio were estimated. The procedure followed the instructions given by a WHO laboratory manual for characterization of T-cell subsets in peripheral blood. ${ }^{17}$

Examination for malaria and intestinal helminths. Examinations for malaria parasites and intestinal helminth eggs were carried out as described previously. ${ }^{10}$

Statistical analysis. Viral loads and infection intensities were normalized by $\log _{10}$ transformation. The two sample Students $t$ test was used to analyze for differences in means, and the $\chi^{2}$ test for differences in prevalences between groups. The effect of DEC treatment on HIV load, CD4\%, and CD4/ CD8 ratio was analyzed according to standard procedures for cross-over trials as described by Altman. ${ }^{18}$ Welch's version of the $t$ test was used to test for treatment effect, period effect, and treatment period interaction. No period effect and no treatment period interaction were observed. A linear model was fitted with the period differences as response and treatment groups, infection status, and their interaction as predictors; a significant interaction term means that the treatment effect differs between the $\mathrm{HIV}^{+}$and $\mathrm{HIV}^{+} / \mathrm{CFA}^{+}$groups.

\section{RESULTS}

Infection status-eligible individuals were recruited from May to June 2002. Collection of baseline data, randomization and the first round of treatment occurred in June 2002. The study participants were followed up at 1, 12, 13, and 24 weeks after the first round of treatment. Cross-over was at 12 weeks after the first treatment. We now describe the baseline characteristics of the HIV-positive study participants (with or without $W$. bancrofti co-infection) and the effect of DEC on HIV load, CD4\%, and CD4/CD8 ratio in these patients during a 12-week period only, as there was no measurable effect of DEC after 1 week. There were no adverse events or side effects related to treatment among the study participants.

Characterization of the study groups. Among the $27 \mathrm{HIV}$ positive study participants, 10 (37\%) were males (Table 1). The mean age was 41 (range 22-70) years, and there was no significant difference in age between males and females. The mean HIV load was $3.71 \log$ units, and the mean CD4\% was 27.3. CFA was present in $12(44 \%)$ individuals, and their mean intensity was 33,287 antigen units. $P$. falciparum was found in 5 individuals (19\%) with a geometric mean intensity of 134 parasite/ $\mu \mathrm{L}$ blood among those infected. Hookworm eggs were found in $13(62 \%)$ of 21 examined individuals (stool samples were not obtained from 6 individuals) with a geometric mean intensity of 410 eggs per gram (epg) of feces among those infected. Eggs from A. lumbricoides and Schistosoma mansoni were not found in any of the study individuals, and T. trichiura eggs were found in 1 only. There was no significant sex difference in the prevalence and intensity of CFA, P. falciparum, or hookworm infection. When the 27 study participants were divided into two groups, with and without filarial co-infection, the prevalence of hookworm was $63.3 \%$ and $60.0 \%(P=0.86)$, respectively, and the prevalence of $P$. falciparum was $27.3 \%$ and $13.3 \%(P=0.37)$, respectively.

Effect of DEC treatment on HIV load. The individual HIV load levels at 12 and 24 weeks follow-up in individuals with $\left(\mathrm{CFA}^{+}\right)$or without $\left(\mathrm{CFA}^{-}\right)$filarial co-infection, are presented in Figure 2. The effect of DEC on viral load was first assessed in all HIV-positive individuals, disregarding filarial status $\left(\mathrm{CFA}^{+} / \mathrm{CFA}^{-}, N=26\right)$, and subsequently in individuals with $\left(\mathrm{CFA}^{+}, N=11\right)$ or without $\left(\mathrm{CFA}^{-}, N=15\right)$ filarial coinfection, separately. One sample could not be amplified in

TABLE 1

Background characteristics of the $27 \mathrm{HIV}$-positive study individuals

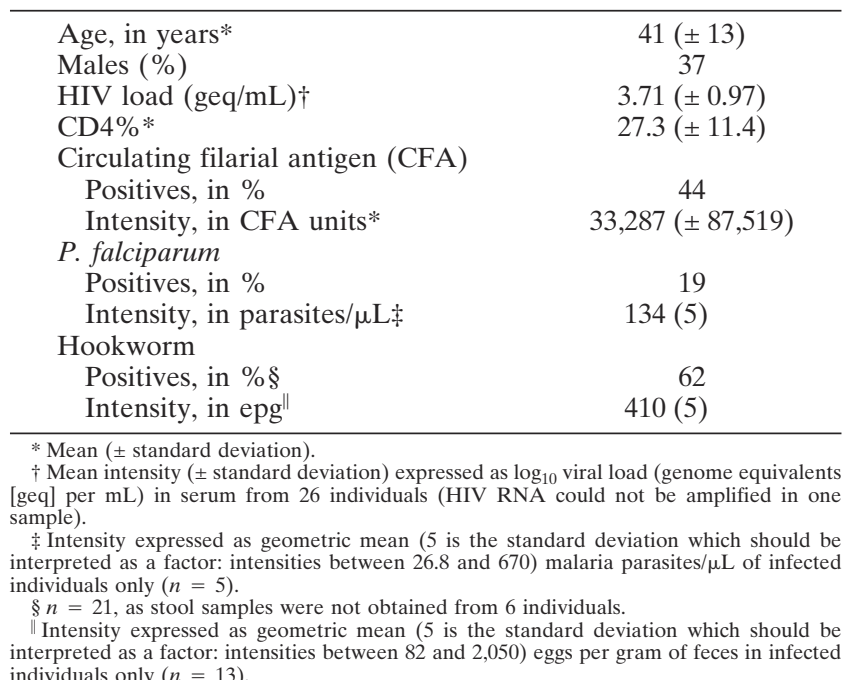



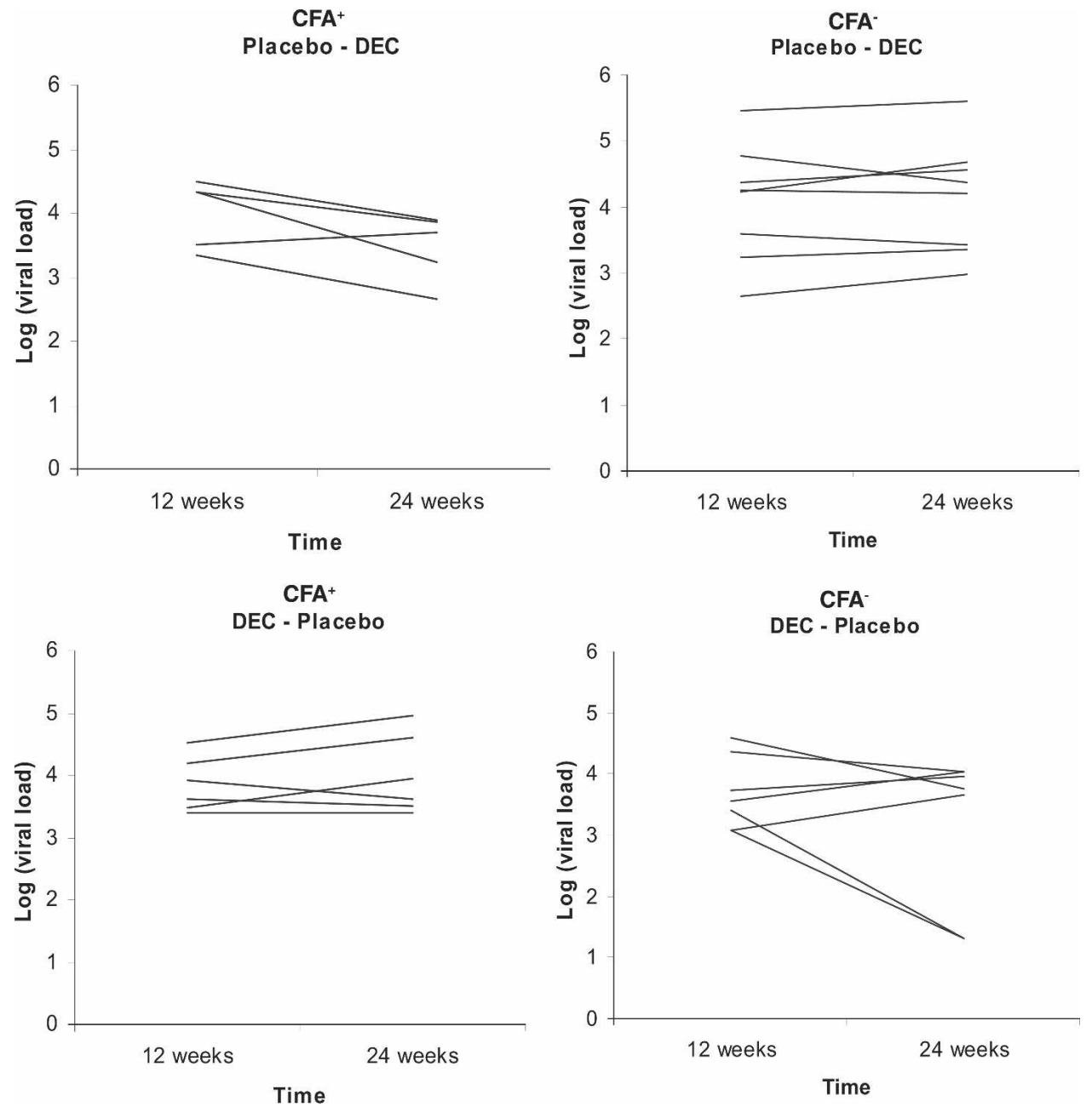

FIGURE 2. Individual HIV load levels in HIV-positive individuals with $\left(\mathrm{CFA}^{+}\right)$or without $\left(\mathrm{CFA}^{-}\right)$W. bancrofti co-infection at 12 and 24 weeks follow-up. The subfigure headings refer to the $W$. bancrofti infection status $\left(\mathrm{CFA}^{+}\right.$or $\left.\mathrm{CFA}^{-}\right)$and the order in which DEC and placebo were given. In 1 sample from the $\mathrm{CFA}^{+}$group, HIV RNA could not be amplified in PCR; therefore this sample was omitted from the HIV load analyses.

PCR but tested positive in 2 different HIV field tests ${ }^{10}$ and was omitted from the HIV load analyses.

The overall analysis among the HIV-positive individuals $\left(\mathrm{CFA}^{+} / \mathrm{CFA}^{-}\right)$showed no significant treatment effect on HIV load $(0.059 \log$ unit increase $\approx 15 \%$ [95\% confidence interval $\{\mathrm{CI}\}:-0.22 ; 0.34], P=0.66)$. However, when the results were stratified by filarial status, an effect was observed in both groups (Figure $3 \mathrm{~A}$ ). In the $\mathrm{CFA}^{+}$group, a significant reduction in HIV load $(-0.34 \log$ unit [95\% CI: $-0.637 ;-0.048]$, $P=0.03$ ), equaling $54 \%$, was observed. In contrast, a $0.36 \mathrm{log}$ unit increase in HIV load (0.36 log unit [95\% CI: -0.10; 0.82], $P=0.11)$, which equals a doubling (2.3 times the pretreatment level), was observed in the $\mathrm{CFA}^{-}$group, but the increase was not statistically significant. When the effect of DEC on HIV load was compared in the $\mathrm{CFA}^{+}$and $\mathrm{CFA}^{-}$ groups, it was shown to be significantly different $(P=0.007)$.

Effect of DEC treatment on the CD4\% and CD4/CD8 ratio. The individual change in $\mathrm{CD} 4 \%$ in the period between the 12- and 24-week follow-up varied considerably (with both increases and decreases observed), and given that day-to-day variation may also occur, these data should be interpreted with caution. The changes in $\mathrm{CD} 4 / \mathrm{CD} 8$ ratio in the same period were very small. Because of these facts, individual changes in $\mathrm{CD} 4 \%$ and $\mathrm{CD} 4 / \mathrm{CD} 8$ ratio are not presented graphically. Instead, the estimated mean effect of DEC on $\mathrm{CD} 4 \%$ and $\mathrm{CD} 4 / \mathrm{CD} 8$ ratio is presented in Figure 3, B and C, respectively. As with HIV load, the effect was first assessed in all HIV-positive individuals $\left(\mathrm{CFA}^{+} / \mathrm{CFA}^{-}, N=27\right)$ and then in those with $\left(\mathrm{CFA}^{+}, N=12\right)$ or without $\left(\mathrm{CFA}^{-}, N=15\right)$ filarial co-infection, separately. The overall analysis among the HIV-positive individuals $\left(\mathrm{CFA}^{+} / \mathrm{CFA}^{-}\right)$showed no significant change in $\mathrm{CD} 4 \%$ (mean increase of $1.20 \%$ point [95\% CI: $-2.24 ; 4.63$ ], $P=0.48$ ) nor in the $\mathrm{CD} 4 / \mathrm{CD} 8$ ratio after treatment (mean increase of $0.08 \%$ point $[95 \% \mathrm{CI}$ : $-0.09 ; 0.25], P=0.36)$.

In the $\mathrm{CFA}^{+}$group, the 12-week treatment effect on the CD $4 \%$ was an increase of $2.5 \%$ points (95\% CI: -4.90 ; 9.94 , $P=0.45)$, but the increase was not statistically significant (Figure 3B). In the $\mathrm{CFA}^{-}$group, an insignificant effect (increase of $0.12 \%$ point $[95 \%$ CI: $-3.41 ; 3.64], P=0.95$ ) was likewise observed. In contrast to HIV load, there was no significant difference in the treatment effect in the two groups $(P=0.48)$.

The effect of DEC treatment on the mean $\mathrm{CD} 4 / \mathrm{CD} 8$ ratio was, as for the $\mathrm{CD} 4 \%$, small and statistically insignificant in both the $\mathrm{CFA}^{+}$and $\mathrm{CFA}^{-}$group with a mean increase in 

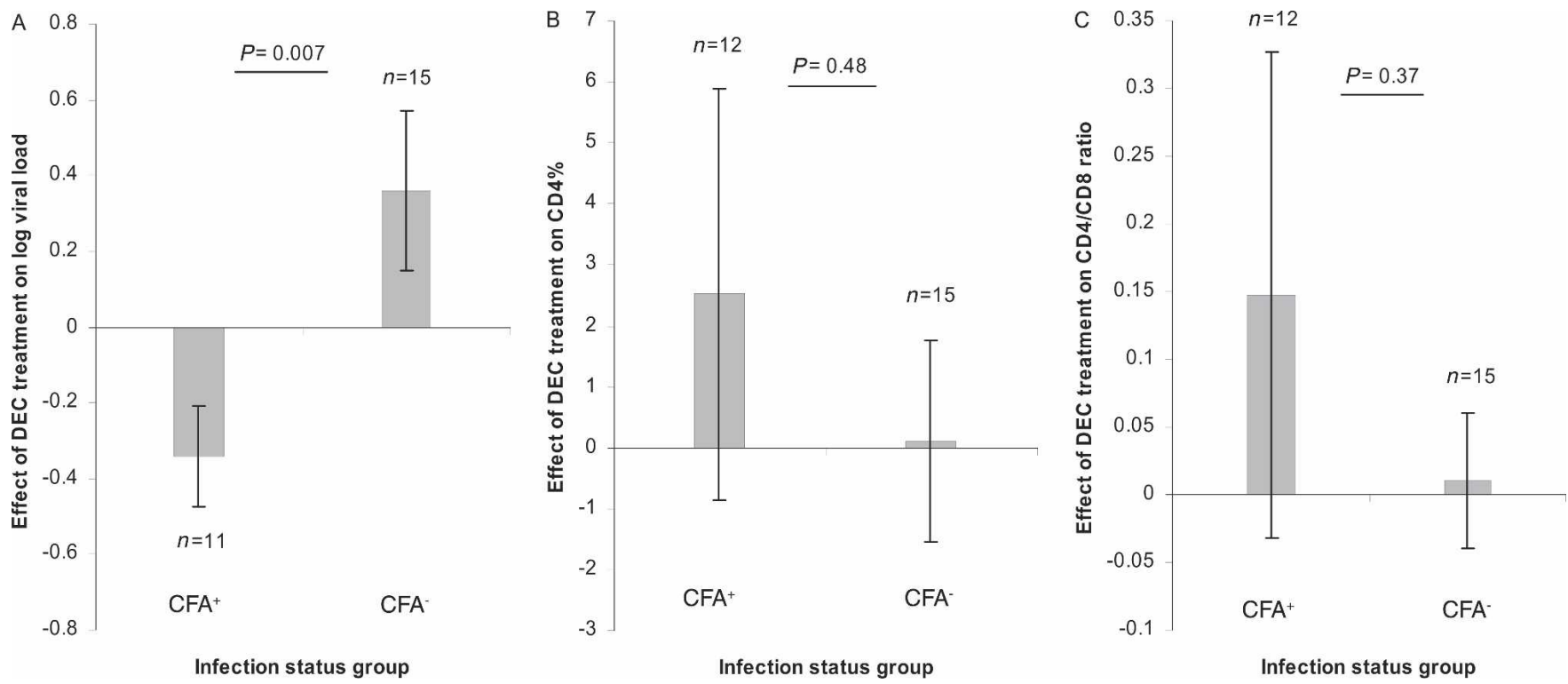

Figure 3. Effect of DEC treatment on HIV load (A), CD4\% (B), and CD4/CD8 ratio (C) in the $\mathrm{CFA}^{+}$and $\mathrm{CFA}^{-}$groups 12 weeks after treatment. Treatment effect is calculated according to the cross-over design as half the difference in the change over time between individuals receiving treatment in the order placebo-DEC and DEC-placebo. Vertical bars represent standard error. In 1 sample from the CFA ${ }^{+}$group, HIV RNA could not be amplified in PCR; therefore this sample was omitted from the HIV load analyses (A).

CD4/CD8 ratio of $0.15 \%$ point $(95 \% \mathrm{CI}:-0.24 ; 0.54, P=$ 0.38 ) and $0.01 \%$ point $(95 \% \mathrm{CI}:-0.10 ; 0.1, P=0.84)$, respectively (Figure 3C). The treatment effect in the 2 groups was not statistically different $(P=0.37)$.

\section{DISCUSSION}

The present study analyzed the effect of DEC treatment on HIV load, CD4\%, and CD4/CD8 ratio over a 12-week followup period in HIV-positive individuals with or without filarial co-infection. Only individuals with asymptomatic infections were included in the study to avoid confounding effects related to immunologic changes in the late stages of HIV infection and in chronic manifestations of filariasis. The pretreatment survey showed a high prevalence of $P$. falciparum malaria and hookworm infection in the study individuals.

The finding of a significant reduction in HIV load in the HIV-positive individuals with filarial co-infection after treatment indicates that DEC treatment reduces viral load in HIV and filariae-positive individuals and may thus slow down the progression of HIV. It seems likely that DEC acts through its effect on the filarial infection. The mechanism behind this is probably immunologically mediated, as treatment of the filarial co-infection may result in a switch from a predominant Th2-type response (known to be associated with filarial infections ${ }^{19-21}$ and infections with other helminths ${ }^{5,22,23}$ ) to an enhanced Th1-type response favorable in the combat against HIV infection. ${ }^{4,24}$ Moreover, the reduction in viral load may be related to a reduced level of immune activation, as antifilarial treatment may cause a reduction in activated $\mathrm{T}$ cells and thereby reduce susceptibility to HIV and delay the viral replication. The contrasting finding of an increase, though not statistically significant, in HIV load among HIV-positive individuals without filarial infection may lead to the thought that DEC could be harmful in terms of increasing HIV load. However, the trend of an increase in CD4\%, rather than a decrease, in the same group of individuals does not lend support to this suspicion.

The difference in the effect of DEC on HIV load in individuals with and without filarial co-infection supports the idea that DEC acts through its antifilaricidial properties rather than through a direct (pharmacodynamic) effect on HIV load in the dose applied in this study. However, it cannot be excluded that administration of DEC in a higher dose and/or for a longer period of time could also have a direct effect on HIV infection. Previous studies in cats have indicated that DEC may have a direct effect on retroviral infections in cats, ${ }^{25,26}$ and DEC treatment $(5 \mathrm{mg} / \mathrm{kg}$ body weight administered for 30 days) of HIV-infected humans in Guatemala intriguingly showed that 5 out of 7 individuals, who tested positive for HIV 1 p24 antigen before treatment, were negative (4 persons) or had a lower (1 person) serum p24 value after 4 weeks of treatment. ${ }^{27}$

The largest increase in CD4\% after treatment was observed in the $\mathrm{CFA}^{+}$group, which corresponds well with the significant decrease in HIV load in this group. The trend of an increase in $\mathrm{CD} 4 \%$, rather than a decrease, in the HIVpositive individuals without filarial infection indicates that DEC had no harmful effect on the health of these patients. It could be speculated that the time required for the treatment effect to be reflected in the CD4\% is longer than the followup period, and the increase in CD4\% after treatment might have been bigger had the patients been followed up for a longer time period. In addition, a higher dose of DEC may have resulted in a more distinct increase in CD4\%.

Generally, the change in the CD4/CD8 ratio after treatment was small. A more pronounced increase in this ratio might have been expected in the group of HIV-positive individuals with filarial co-infection after antifilarial treatment as a result of the increased CD4\%. However, because production and activity of cytotoxic T cells (CD8 cells) appear to be associated with a Th1-type response, ${ }^{5}$ an increase in the CD4/ 
CD8 ratio may have failed to appear because the increase in CD4 cells was accompanied by a simultaneous increase in production of CD8 cells as a result of a switch from a Th2- to a Th1-predominated response after treatment of the filariae.

In summary, the presented data suggest that DEC treatment may have an important positive impact on the host's ability to respond to the HIV infection in individuals coinfected with filariae and may thereby slow down the progression of HIV. The ongoing global efforts to control lymphatic filariasis, which in many areas rely on annual mass treatment with DEC, may thus have an additional beneficial effect in terms of contributing to alleviation of the HIV/AIDS epidemic in areas where HIV and lymphatic filariasis co-exist. A positive effect of DEC on HIV in W. bancrofti-endemic areas may be even more pronounced if the drug was administered at a higher dose and/or more frequently than once a year, a perspective that deserves to be further investigated.

The prospects of DEC, being an inexpensive and welltolerated drug, as a tool in the combat against HIV/AIDS may be even wider. If studies similar to the present study but with 1) a higher dose of DEC, 2) continuous or regular administration (contrary to the single dose in the present study), 3) larger study populations, and 4) longer follow-up periods could provide indications of an anti-retroviral effect of DEC, these might form the basis for development of new DECbased treatment strategies to combat HIV/AIDS. Studies on the anti-retroviral efficacy of DEC (or DEC derivatives) would also be of importance in attempts to be at the forefront of potential development of resistance to the currently used preparations.

Received August 16, 2006. Accepted for publication April 18, 2007.

Acknowledgments: The study participants are gratefully acknowledged for their collaboration, and the technical staff at Bombo Research Station (the late Joyce Kivugo, Sudi Hassani, Chille Malimi, Charles Guzo, Zaina Maumba, and Mwanaidi Bagabuje) and DBLCentre for Health Research and Development (Bente Larsen Jensen and Benedikte Løhr Wilken) are thanked for their valuable assistance throughout the study. We are grateful to the Tanga AIDS Working Group (Firmina S. Mberesero, Anna Chaze, and Ole Sabaini) for providing professional HIV counseling to the study participants. The National Institute for Medical Research, Dar es Salaam (Mwele Malecela-Lazaro), is thanked for collaboration and support. Financial support: The research was funded by the Council for Development Research, DBL-Centre for Health Research and Development, the AIDS Foundation, the Wedell-Wedellsborg Foundation, and the Knud Højgaard Foundation, all in Denmark.

Disclaimer: The paper is published with permission of the Director General, National Institute for Medical Research, Tanzania.

Authors' addresses: Nina O. Nielsen, Paul E. Simonsen, and Pascal Magnussen, DBL - Centre for Health Research and Development, Department of Veterinary Pathobiology, Faculty of Life Sciences, University of Copenhagen, Jægersborg Allé 1D, 2920 Charlottenlund, Denmark, Telephone: +45 773277 32, Fax: +45 773277 33, E-mail: nnielsen@dblnet.dk. Peter Dalgaard, Department of Biostatistics, University of Copenhagen, Oester Farimagsgade 5, P.O. Box 2099, 1014 Copenhagen K, Denmark, Telephone: +45 353279 18, Fax: +45 353279 07, E-mail: p.dalgaard@biostat.ku.dk. Henrik Krarup, Department of Clinical Biochemistry, Aalborg University Hospital, P.O. Box 561, 9100 Aalborg, Denmark, Telephone: +45 99 3211 11, Fax: +45-98 1330 60, E-mail: h.krarup@rn.dk. Stephen Magesa, National Institute for Medical Research, Ubwari Research Station, P.O. Box 81, Muheza, Tanzania, Telephone: +255-272641132, Fax: +255-27-2643869, E-mail: smagesa@nimr.or.tz. Henrik Friis, Department of Human Nutrition, University of Copenhagen, Rolighedsvej 30, 1958 Frederiksberg, Denmark, Telephone: +45 3528 38 70, Fax: +45 352824 83, E-mail: hfr@life.ku.dk.
Reprint requests: Nina O. Nielsen, DBL-Centre for Health Research and Development, Department of Veterinary Pathobiology, Faculty of Life Sciences, University of Copenhagen, Jægersborg Allé 1D, 2920 Charlottenlund, Denmark, E-mail: nnielsen@dblnet.dk.

\section{REFERENCES}

1. Borkow G, Bentwich Z, 2004. Chronic immune activation associated with chronic helminthic and human immunodeficiency virus infections: role of hyporesponsiveness and anergy. Clin Microbiol Rev 17: 1012-1030.

2. Bentwich Z, Maartens G, Torten D, Lal AA, Lal RB, 2000. Concurrent infections and HIV pathogenesis. AIDS 14: 2071-2081.

3. Bentwich Z, Kalinkovich A, Weisman Z, 1995. Immune activation is a dominant factor in the pathogenesis of African AIDS. Immunol Today 16: 187-191.

4. Actor JK, Shirai M, Kullberg MC, Buller RML, Sher A, Berzofsky JA, 1993. Helminth infection results in decreased virusspecific $\mathrm{CD}^{+}$cytotoxic T-cell and Th1 cytokine responses as well as delayed virus clearance. Proc Natl Acad Sci USA 90: 948-952.

5. Maggi E, Mazzetti M, Ravina A, Annunziato F, De Carli M, Piccinni MP, Manetti R, Carbonari M, Pesce AM, Del Prete G, Romagnani S, 1994. Ability of HIV to promote a Th1 to Th0 shift and to replicate preferentially in Th2 and Th0 Cells. Science 265: 244-248.

6. Fincham JE, Markus MB, Mansvelt EPG, 1999. Could nonselective anthelmintic treatment programmes contribute to control of the spread of HIV infection and AIDS? Trans $R$ Soc Trop Med Hyg 93: 536.

7. Wolday D, Mayaan S, Mariam ZG, Berhe N, Seboxa T, Britton S, Galai N, Landay A, Bentwich Z, 2002. Treatment of intestinal worms is associated with decreased HIV plasma viral load. J Acquir Immune Defic Syndr 31: 56-62.

8. Brown M, Kizza M, Watera C, Quigley MA, Rowland S, Hughes $\mathrm{P}$, Whitworth JAG, Elliot AM, 2004. Helminth infection is not associated with faster progression of HIV disease in coinfected adults in Uganda. J Infect Dis 190: 1869-1879.

9. Abu-Raddad LJ, Patnaik P, Kublin JG, 2006. Dual infection with HIV and malaria fuels the spread of both diseases in subSaharan Africa [published correction appears in Science 315: 598]. Science 314: 1603-1606.

10. Nielsen NO, Simonsen PE, Magnussen P, Magesa S, Friis H, 2006. Cross-sectional relationship between HIV, lymphatic filariasis and other parasite infections in adults in coastal northeastern Tanzania. Trans R Soc Trop Med Hyg 100: 543-550.

11. Nielsen NO, Friis H, Magnussen P, Krarup H, Magesa S, Simonsen PE, 2007. Co-infection with sub-clinical HIV and Wuchereria bancrofti, and the role of malaria and hookworms, in adult Tanzanians: infection intensities, CD4/CD8 counts and cytokine responses. Trans $R$ Soc Trop Med Hyg 101: 602-612.

12. Vaughan JP, Morrow RH, 1989. Manual of Epidemiology for District Health Management. Geneva: World Health Organization, $178 \mathrm{pp}$.

13. Krarup HB, Drewes AM, Madsen H, 1998. A quantitative HCVPCR test for routine diagnostics. Scand J Clin Lab Invest 58: 415-422.

14. Erber WN, Pinching AJ, Mason DY, 1984. Immunocytochemical detection of $\mathrm{T}$ and $\mathrm{B}$ cell populations in routine blood smears. Lancet 1: 1042-1046.

15. Lisse IM, Whittle H, Aaby P, Normark M, Gyhrs A, Rayder LP, 1990. Labelling of T-cell subsets under field conditions in tropical countries: adaptation of the immuno-alkaline phosphatase staining method for blood smears. J Immunol Methods 129: $49-53$.

16. Lisse IM, Böttiger B, Christensen LB, Knudsen K, Aaby P, Gottschau A, Urassa W, Mhalu F, Biberfeld G, Brattegaard K, Diallo K, N'Gom PT, Whittle H, 1997. Evaluation of T cell subsets by an immunocytochemical method compared to flow cytometry in four countries. Scand J Immunol 45: 637-644.

17. WHO, 1993. Laboratory Manual for a Simple Method for Characterization of T-Cell Subsets in Peripheral Blood: An Immuno-Alkaline Phosphatase Method. Review 1: Global Pro- 
gramme on AIDS. DIA/MISC/92.2. Geneva: World Health Organization.

18. Altman DG, 1999. Practical Statistics for Medical Research. London: Chapman \& Hall, 467-471.

19. King CL, Mahanty S, Kuramaswami V, Abrahams JS, Regunathan J, Jayaraman K, Ottesen EA, Nutman TB, 1993. Cytokine control of parasite anergy in human lymphatic filariasis. J Clin Invest 92: 1667-1673.

20. Yazdanbakhsh M, Sartono E, Kruize YCM, Kurniawan A, van der Pouw-Kraan T, van der Meide PH, Selkirk ME, Hintzen RQ, Partono F, van Lier RAW, Maizels RM, 1993. Elevated levels of $\mathrm{T}$ cell activation antigen CD27 and increased interleukin-4 production in human lymphatic filariasis. Eur J Immunol 23: 3312-3317.

21. Sartono E, Kruize YCM, Kurniawan A, Maizels RM, Yazdanbakhsh M, 1996. In Th2 biased lymphatic filariasis patients, responses to purified protein derivative of Mycobacterium tuberculosis remain Th1. Eur J Immunol 26: 501-504.

22. Borkow G, Bentwich Z, 2000. Eradication of helminth infections may be essential for successful vaccination against HIV and tuberculosis. Bull World Health Organ 78: 1368-1369.
23. Yazdanbakhsh M, van den Biggelaar A, Maizels RM, 2001. Th2 responses without atopy: immunoregulation in chronic helminth infections and reduced allergic disease. Trends Immunol 22: $372-377$.

24. Clerici M, Shearer GM, 1993. A Th1 $\rightarrow$ Th2 switch is a critical step in the etiology of HIV infection. Immunol Today 14: 107-110.

25. Kitchen LW, Mather FJ, Cotter SM, 1988. Effect of continuous oral diethylcarbamazine treatment on lymphocyte counts of feline leukaemia virus-infected cats. J Clin Lab Immunol 27: $179-181$.

26. Kitchen LW, Cotter SM, 1988. Effect of diethylcarbamazine in serum antibody to feline oncornavirus-associated cell membrane antigen in feline leukaemia virus cats. J Clin Lab Immunol 25: 101-103.

27. Arathoon EG, Mejia CR, Estrada y Martin RM, Hernandez JE, Turner GJ, Kitchen LW, 1994. Serum HIV 1 p24 levels and body weight measurements before and after 4 weeks of diethylcarbamazine treatment given to HIV 1 seropositive persons. Int J Antimicrob Agents 3: 275-278. 